\title{
Dissociable Contributions of Anterior Cingulate Cortex and Basolateral Amygdala on a Rodent Cost/Benefit Decision-Making Task of Cognitive Effort
}

\author{
Jay G Hosking*,', Paul J Cocker' and Catharine A Winstanley*,' \\ 'Department of Psychology, University of British Columbia, Vancouver, BC, Canada
}

\begin{abstract}
Personal success often requires the choice to expend greater effort for larger rewards, and deficits in such effortful decision making accompany a number of illnesses including depression, schizophrenia, and attention-deficit/hyperactivity disorder. Animal models have implicated brain regions such as the basolateral amygdala (BLA) and anterior cingulate cortex (ACC) in physical effort-based choice, but disentangling the unique contributions of these two regions has proven difficult, and effort demands in industrialized society are predominantly cognitive in nature. Here we utilize the rodent cognitive effort task (rCET), a modification of the five-choice serial reactiontime task, wherein animals can choose to expend greater visuospatial attention to obtain larger sucrose rewards. Temporary inactivation (via baclofen-muscimol) of BLA and ACC showed dissociable effects: BLA inactivation caused hard-working rats to 'slack off and 'slacker' rats to work harder, whereas ACC inactivation caused all animals to reduce willingness to expend mental effort. Furthermore, BLA inactivation increased the time needed to make choices, whereas ACC inactivation increased motor impulsivity. These data illuminate unique contributions of BLA and ACC to effort-based decision making, and imply overlapping yet distinct circuitry for cognitive vs physical effort. Our understanding of effortful decision making may therefore require expanding our models beyond purely physical costs. Neuropsychopharmacology (2014) 39, I558-1567; doi:I0.1038/npp.2014.27; published online 26 February 2014
\end{abstract}

Keywords: animal models; choice; individual differences; mental effort; neural circuitry

\section{INTRODUCTION}

Attaining many of the goals we seek requires the decision to invest valuable resources such as time and effort. Diminished motivation for such rewards can negatively impact an individual's quality of life, reaching clinical significance in a number of conditions including schizophrenia, depression, and attention-deficit/hyperactivity disorder (ADHD; Egeland et al, 2010; Gold et al, 2013; Hammar et al, 2011).

The amygdala and anterior cingulate cortex (ACC) are two critical brain regions in decision making. Patients with damage to either region demonstrate impairments on laboratory models of 'real-world' decision making where levels of reward and cost are varied across options (Bechara et al, 1999; Brand et al, 2007; Manes et al, 2002; van Honk et al, 2013). Similarly, functional neuroimaging and intracranial electrophysiology suggest that the amygdala and ACC are involved in evaluating costs and rewards to guide subsequent behavior (Basten et al, 2010; Jenison et al, 2011; Smith et al, 2009; Williams et al, 2004), and internally

* Correspondence: JG Hosking or Dr CA Winstanley, Department of Psychology, University of British Columbia, 2136 West Mall, Room 35 I0, Vancouver, BC V6T IZ4, Canada, Tel: + I 604827 5083, Fax: + 604822 6923, E-mail: jayhosking@psych.ubc.ca or cwinstanley@psych.ubc.ca

Received 18 October 2013; revised 10 January 2014; accepted 28 January 2014; accepted article preview online 5 February 2014 generated changes in choice correlate with amygdala and ACC activity (Sokol-Hessner et al, 2013; Walton et al, 2004).

The importance of these regions has also been evaluated in animal models of effort-based decision making, wherein animals can choose to obtain larger rewards by making more responses on a lever or climbing a barrier (Floresco et al, 2008b; Salamone et al, 1994). Excitotoxic lesions and pharmacological inactivation of either the basolateral nucleus of the amygdala (BLA) or ACC shifts rats' behavior away from high-effort, high-reward (HR) options (GhodsSharifi et al, 2009; Rudebeck et al, 2006b), as does contralateral disconnection of these regions (Floresco and Ghods-Sharifi, 2007). Thus, the BLA and ACC are necessary to overcome aversive work requirements and select the maximally rewarding option, although their unique contributions remain unclear.

However, decision costs are not unitary in their underlying circuitry (Floresco et al, 2008a). Although animal models vary physical work requirements across options, effort costs in industrialized society are overwhelmingly cognitive in nature; literature on human effort reflects this (Croxson et al, 2009; Kool et al, 2010; Naccache et al, 2005). Broadly speaking, cognitive or mental effort costs are those that are non-physical in nature and tax limited neurobiological resources, as observed through the psychological constructs of working memory, attention, response inhibition, etc. (Schmidt et al, 2012). Regions deemed inessential 


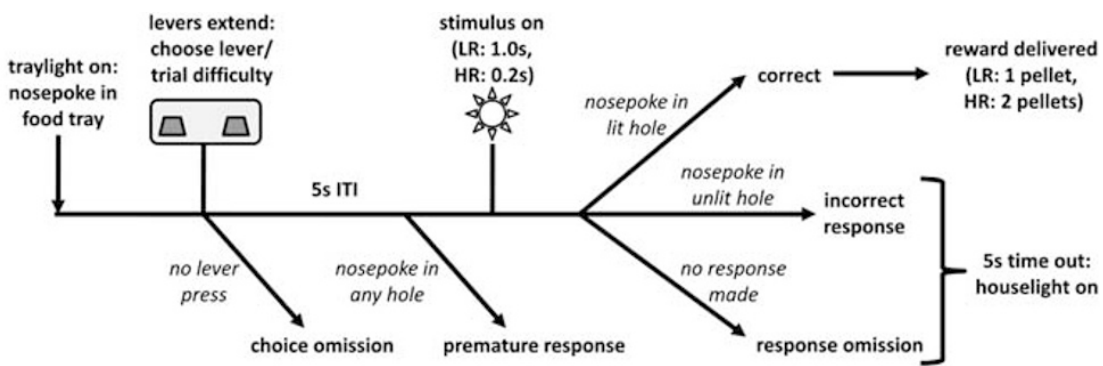

Figure I Schematic of a trial in the rodent cognitive effort task. Trials began with illumination of the food tray light. A nosepoke response in the food tray extinguished the light, commencing a new trial and extending the levers. Each lever was permanently designated to initiate either low-effort/LR or high-effort/ HR trials. If one of the two levers was pressed, both levers retracted and a 5-s ITI would begin. Following the ITI, one of five stimulus lights would briefly illuminate, $1.0 \mathrm{~s}$ for a LR trial and $0.2 \mathrm{~s}$ for a HR trial. A nosepoke response in the illuminated aperture (ie, a correct response) led to a sugar reward, one pellet for a LR trial and two pellets for a HR trial. The food tray light would then illuminate again, indicating the opportunity to start the subsequent trial. A number of behaviors led to a 5-s time-out, signaled by house-light illumination: failure to make a lever response (choice omission); failure to withhold responding during the ITI (premature response); nosepoke in an unlit hole following the stimulus (incorrect response); failure to make a nosepoke response following the stimulus (response omission). Figure reprinted with permission from Cocker et al (20/2).

in physical effort (eg, the prefrontal cortex; Walton et al, $2003 \mathrm{~b}$ ) have been shown to have a prominent role in human cognitive effort (McGuire and Botvinick, 2010; Schmidt et al, 2012). Furthermore, these human studies have emphasized how individual differences in brain function influence individual differences in effort expenditure (Treadway et al, 2012b), an examination all but absent from animal effort literature (but see Randall et al, 2012).

Our group has recently validated a rodent cognitive effort task (rCET), wherein animals can choose between loweffort/low-reward (LR) and high-effort/HR options, and show that individual differences in animals' sensitivity to cognitive effort mediates the effects of psychostimulants (Cocker et al, 2012), results that were distinct from animal models of physical effort (Floresco et al, 2008b). In the rCET, a stimulus light is presented at pseudorandom in one of five apertures, and the animal must nosepoke in that aperture for reward. Cognitive effort costs are varied by the amount of visuospatial attention required in a given trial: when an easy (LR) trial is chosen, the light remains illuminated for a lengthy $1.0 \mathrm{~s}$ and little attention is required to identify the aperture; when a hard (HR) trial is chosen, the light is illuminated for only $0.2 \mathrm{~s}$, making the aperture much more difficult to identify (as reflected by lower task accuracy, ie, performance).

The goal of this study was therefore to use the rCET to determine the importance of the BLA and ACC in the societally relevant construct of cognitive effort, whether the impact of silencing these regions depends on individual differences in willingness to work, and thus the unique contributions of these regions to effortful decision making.

\section{MATERIALS AND METHODS}

\section{Subjects}

Subjects were 56 male Long-Evans rats (Charles River Laboratories, St Constant, QC, Canada), in two cohorts (BLA: $n=24$, and ACC: $n=32$ ), weighing $275-300 \mathrm{~g}$ at the beginning of the experiment. Animals were maintained at $\sim 85 \%$ of their free-feeding weight and food restricted to $14 \mathrm{~g}$ rat chow per day. Water was available ad libitum. Animals were pair-housed in a climate-controlled colony room on a 12-h reverse light-dark cycle (lights off: 0800 hours; temperature: $21^{\circ} \mathrm{C}$ ). All housing and testing was in accordance with the Canadian Council of Animal Care, and all procedures were approved by the UBC Animal Care Committee.

\section{The rCET Training and Testing}

Testing took place within 16 standard five-hole operant chambers, each supplemented with two retractable levers and enclosed in a ventilated, sound-attenuating cabinet (Med Associates, VT, USA; for a detailed description, see Winstanley et al, 2010). The chambers were controlled by software written in Med-PC by CAW, running on an IBMcompatible computer.

Habituation and pre-task training have been previously described (see Cocker et al, 2012, including Supplementary Methods). Briefly, animals learned to make a nosepoke response in an illuminated aperture within $5 \mathrm{~s}$ to obtain reward, as per five-choice serial reaction-time task (5CSRTT) training (Winstanley et al, 2010). In subsequent sessions, animals were trained to respond on two retractable levers at a fixed ratio 1 schedule for reward. Animals were then trained on a forced-choice variant of the rCET (40-50 sessions), wherein only a single lever extended per trial, before the standard free-choice program.

The design of the rCET has been previously described (Cocker et al, 2012) and a schematic of the trial structure and subsequent reinforcement is provided in Figure 1. In brief, animals were tested 4-5 days per week in 30-min sessions of no fixed trial limit. At the outset of training, the levers were permanently designated to initiate either loweffort/LR or high-effort/HR trials, and these designations were evenly counterbalanced across subjects. A trial began with a nosepoke at the food tray when the light inside was illuminated, thereby extending the levers. If one of the levers was pressed, thus setting the trial as either LR or HR, both levers would retract and a 5-s inter-trial interval (ITI) would begin. Following the ITI, one of the five stimulus lights would be briefly illuminated, with a stimulus duration of $1.0 \mathrm{~s}$ for a LR trial and $0.2 \mathrm{~s}$ for a HR trial. Animals then had $5 \mathrm{~s}$ to nosepoke within the previously illuminated aperture (a correct response) for reward. Animals were rewarded with one sugar pellet for a correct 
LR trial and two sugar pellets for a correct HR trial, and the tray light would again illuminate to signal the opportunity to start the next trial.

Trials went unrewarded for a number of reasons: if animals failed to make a lever response within $10 \mathrm{~s}$ (a choice omission); if animals nosepoked during the ITI (a premature response, a long-used behavioral measure of motor impulsivity; see Robbins, 2002); if animals nosepoked in any aperture other than the one that was illuminated (an incorrect response); and if animals failed to nosepoke at the array within $5 \mathrm{~s}$ after stimulus light illumination (a response omission). All such behaviors were punished with a 5-s time-out period, accompanied by illumination of the house light, during which new trials could not be initiated and thus reward could not be earned. Following a time-out, the tray light was illuminated to signal that the rat could begin the next trial.

\section{Behavioral Measurements}

To minimize the influence of variation in the number of trials completed, choice of HR trials was calculated as a percentage: (number of choices of a particular lever/number of total choices $) \times 100$. In addition, each animal's completed trials were divided into quartiles and the percentage choice of HR trials was measured in these four bins. The following variables were analyzed separately for $\mathrm{LR}$ and $\mathrm{HR}$ trials: accuracy (number of correct responses/number of total responses made) $\times 100)$; nosepoke response omissions ((number of trials omitted/number of correct, incorrect and omitted trials $) \times 100)$; premature responses ((number of premature responses/total number of trials initiated $) \times 100$ ); average latency to choose between the LR and HR levers (lever choice latency); average latency to correctly nosepoke (correct latency); average latency to collect reward (collection latency). The total number of failures to choose a lever at the beginning of a trial (choice omissions) and total number of completed trials was also analyzed. Although many of these behavioral measures initially appear superfluous to the central question of cognitive effort processing, they allow for the elimination of potential confounds in the interpretation of choice behavior.

\section{Surgery}

Surgery and microinfusion procedures were modeled after Floresco and Ghods-Sharifi (2007) and Ghods-Sharifi et al (2009). When baseline performance was deemed statistically stable (30-35 sessions), animals were implanted with 22-gauge stainless steel guide cannulae (Plastics One; Roanoke, VA, USA) bilaterally into the BLA or ACC using standard stereotaxic techniques. Animals in the first cohort were anesthetized with $100 \mathrm{mg} / \mathrm{kg}$ ketamine and $10 \mathrm{mg} / \mathrm{kg}$ xylazine and implanted with BLA cannulae (incisor bar set to $\sim-3.3$ (flat skull): anteroposterior $(\mathrm{AP})=-3.1 \mathrm{~mm}$, mediolateral $(\mathrm{ML})= \pm 5.2 \mathrm{~mm}$ from bregma; dorsoventral $(\mathrm{DV})=$ $-6.5 \mathrm{~mm}$ from dura). Those in the second cohort were anesthetized with $2 \%$ isoflurane in $\mathrm{O}_{2}$ and implanted with ACC cannulae (flat skull: $\mathrm{AP}=+2.0 \mathrm{~mm}, \mathrm{ML}= \pm 0.7 \mathrm{~mm}$ from bregma; $\mathrm{DV}=-1.2 \mathrm{~mm}$ from dura; Paxinos and Watson, 1998). All animals were provided with pre- and post-operative analgesia to minimize pain or discomfort via $5 \mathrm{mg} / \mathrm{kg}$ Anafen.
Guide cannulae were fixed to the skull via three stainless steel screws and dental acrylic, and 29-gauge obdurators with dust caps were inserted and extended flush with the end of the cannulae. Animals were given at least one week of recovery in their home cages before subsequent testing. Twelve animals were excluded because of poor recovery.

\section{Microinfusion}

Following recovery, animals performed 10 free-choice sessions, after which all individuals displayed stable behavior (ie, no effect of session on repeated-measures ANOVA for choice, accuracy, and premature responding over the last three sessions; see 'Data analysis', below). Animals were then habituated to the microinfusion process with two mock infusions, wherein the 30 -gauge injectors with tips extending $1 \mathrm{~mm}$ beyond the guide cannulae were inserted for $2 \mathrm{~min}$ but no infusion was performed, followed by a testing session. Infusions followed a 3-day cycle starting with a baseline session, followed by a drug or saline injection session, and then by a non-testing day; a single 30 -min testing session was performed for each saline and inactivation condition. The BLA and ACC were inactivated by a mixture of the $\mathrm{GABA}_{\mathrm{B}}$ agonist baclofen and the $\mathrm{GABA}_{\mathrm{A}}$ agonist muscimol (SigmaAldrich; Oakville, ON, Canada), prepared separately at $0.5 \mu \mathrm{g} / \mu \mathrm{l}$ in saline and mixed together in equal volumes to form a $0.25 \mu \mathrm{g} / \mu \mathrm{l}$ solution. In all, $0.5 \mu \mathrm{l}$ injections of saline or baclofen-muscimol (ie, $0.125 \mu \mathrm{g}$ of drug) were administered bilaterally at a rate of $0.4 \mu \mathrm{l} / \mathrm{min}$, and injectors were left in place for an additional minute to allow diffusion. Injectors were then removed, obdurators were replaced, and animals were returned to their home cages for $10 \mathrm{~min}$ before being placed in the operant chambers and performing the rCET. On the first infusion day, half of the rats received saline infusions while the other half received baclofen-muscimol; these administrations were reversed on the second infusion day, allowing for a within-subjects comparison.

\section{Histology}

Following completion of all behavioral testing, animals were killed by carbon dioxide exposure. Brains were removed and fixed in $4 \%$ formaldehyde for at least $24 \mathrm{~h}$, transferred to a $30 \%$ sucrose solution, and then frozen and cut via cryostat into $40 \mu \mathrm{m}$ coronal sections. These sections were stained with Cresyl violet for visualization, and the projected locations of the injector tips protruding from the guide cannulae were mapped onto standard sections from Paxinos and Watson (1998).

\section{Data Analysis}

All data were analyzed using within-subjects repeatedmeasures ANOVA in SPSS (version 16.0; SPSS/IBM, Chicago, IL, USA), with choice (two levels: LR or HR) and inactivation (two levels: saline or drug) as repeatedmeasures factors. All percentages were arcsine transformed to minimize artificial ceiling effects (Winstanley et al, 2003; Zeeb et al, 2009) before analysis. Animals were grouped as 'workers' if they chose HR for $>70 \%$ of trials (BLA: $n=9$; ACC: $n=10$ ) and 'slackers' if they chose HR for $\leqslant 70 \%$ of trials (BLA: $n=7$; ACC: $n=18$ ) at post-surgical baseline. 
a $1.70 \mathrm{~mm}$

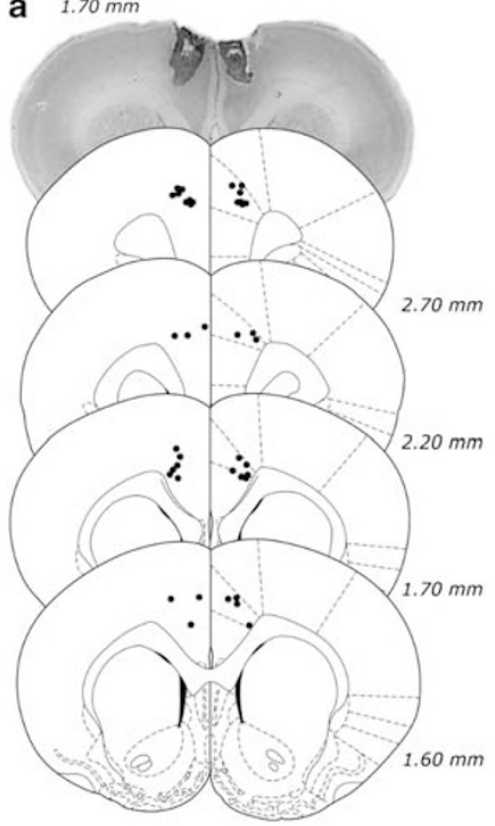

b

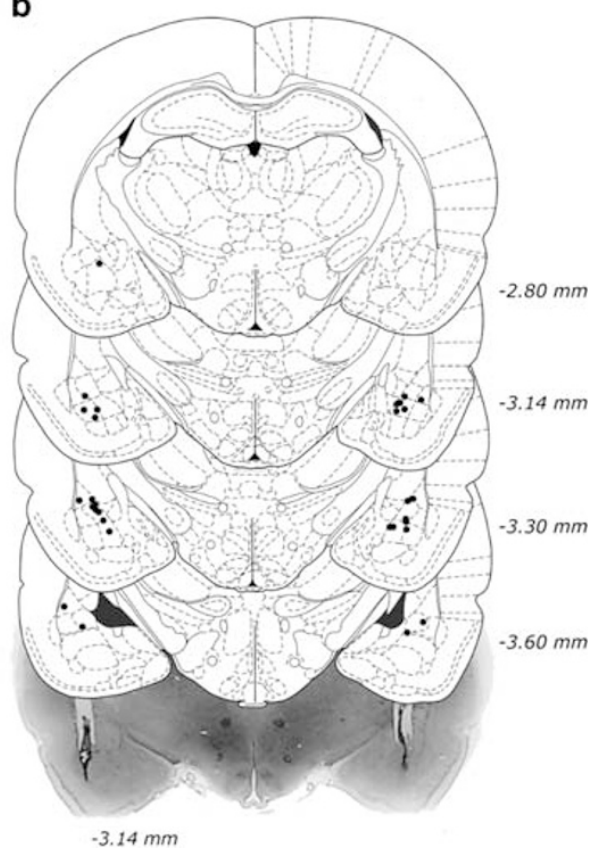

Figure 2 Histological analysis of cannulae implantation. (a) Location of all acceptable ACC infusions, including a representative photomicrograph. (b) Location of all acceptable BLA infusions, including a representative photomicrograph. Coordinates are relative to bregma. Plates modified from Paxinos and Watson (1998).

This subdivision was based on the mean split from the original rCET paper (Cocker et al, 2012), where workers and slackers were categorized based on their preference for greater than or less than the average of $70 \%$ HR trials. To maintain consistency when discussing individual differences and to avoid arbitrary categorization, we therefore held the worker/slacker distinction at $70 \% \mathrm{HR}$ trials for this study. It should be noted that the group averages of HR trials were $69 \%$ and $56 \%$ for BLA and ACC rats, respectively. Furthermore, choice preference was stable throughout the experiment; animals maintained their worker/slacker distinction across post-surgical and saline sessions (session: all Fs $<2.092$, NS). Thus, group (two levels: workers or slackers) was used as a between-subjects factor in all analyses. The completed trials' quartiles (four levels: Q1-Q4) were used as a within-subjects factor for examining choice of HR trials across the session. Any significant effects $(p<0.05)$ were further analyzed via posthoc one-way ANOVA.

\section{RESULTS}

\section{Cannula Placements}

The locations of all acceptable placements, as well as representative samples of BLA and ACC cannulation, are depicted in Figure 2. Two animals from the BLA group and two animals from the ACC group were excluded because of inaccurate placements in one hemisphere, leaving a total of 40 animals for analysis (BLA: $n=14$; ACC: $n=26$ ).

\section{BLA Inactivation}

Choice behavior, accuracy, and premature responses. As per baseline conditions and previous cohorts (Cocker et al,
2012), animals chose high-effort/HR trials more than loweffort/LR trials (choice: $\mathrm{F}_{1,12}=11.144, p=0.006$ ) when the BLA was infused with saline. However, there was substantial individual variation across the group and the previously established worker/slacker distinction held; workers in fact chose significantly more $\mathrm{HR}$ trials than slackers (group: $\mathrm{F}_{1,12}=34.549, p<0.001$ ). Inactivation of the BLA had no effect on animals' choice of LR or HR trials when considered as a homogenous group (inactivation: $\mathrm{F}_{1,12}<0.001$, NS). However, BLA inactivation differentially affected workers and slackers, increasing choice of $\mathrm{HR}$ trials in slackers but decreasing this behavior in workers (Figure 3a; inactivation $\times$ group: $F_{1,12}=5.445$, $p=0.038$ ). Choice effects of BLA inactivation were present from the first quartile of trials, with opposing effects on workers vs slackers predominantly in Q1 and Q4 (inactivation: $\mathrm{F}_{1,12}=0.128$, NS; inactivation $\times$ group: $\mathrm{F}_{1,12}=4.617, \quad p=0.053$; inactivation $\times$ quartile: $\mathrm{F}_{3,12}=3.486, \quad p=0.026 ; \quad$ inactivation $\times$ group $\times$ quartile: $\mathrm{F}_{3,12}=6.848, \quad p=0.001 ; \quad \mathrm{Q} 1$ only-inactivation $\times$ group: $\mathrm{F}_{1,12}=7.768, \quad p=0.016 ; \quad \mathrm{Q} 4$ only-inactivation $\times$ group: $\mathrm{F}_{1,12}=11.939, p=0.005 ; \mathrm{Q} 2 / \mathrm{Q} 3$-inactivation $\times$ group: all Fs $<0.687$, NS).

As expected, animals were more accurate on LR vs HR trials, as shown by a main effect of choice on repeatedmeasures ANOVA (choice: $\mathrm{F}_{1,12}=29.975, p<0.001$ ). Despite its effects on choice, BLA inactivation did not affect accuracy or premature responding (Figures $3 b$ and $c$; inactivation/choice $\times$ inactivation: all Fs $<1.154$, NS). Identical to previous cohorts, there were no differences in accuracy or premature responding between workers and slackers, indicating that choice preferences were not primarily because of individuals' ability to perform the task (group/inactivation $\times$ group: all Fs $<1.012$, NS). 
BLA
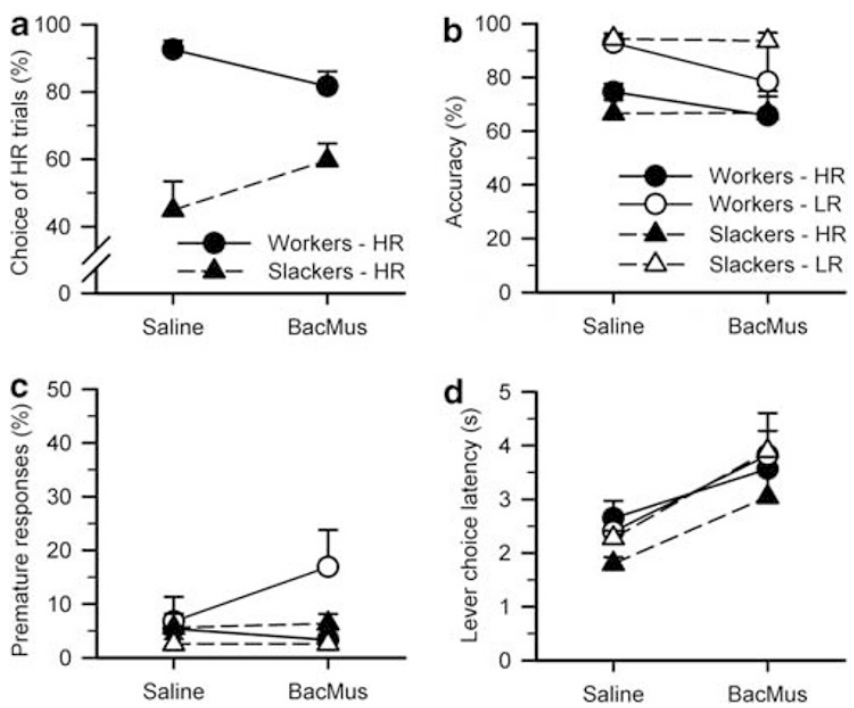

Figure 3 Effects of BLA inactivations on the rodent cognitive effort task. (a) Infusion of baclofen-muscimol into the BLA had no effect on animals' choice of LR or HR trials when considered as a homogenous group (inactivation: $F_{1,12}<0.00 \mathrm{I}, \mathrm{NS}$ ). However, BLA inactivation differentially affected workers and slackers, increasing choice of HR trials in slackers but decreasing this behavior in workers (inactivation $\times$ group: $F_{1,12}=5.445$, $p=0.038)$. ( $b, c)$ Inactivation of the BLA did not affect accuracy or premature responding (inactivation/choice $\times$ inactivation: all $\mathrm{Fs}<\mathrm{I} . \mathrm{I} 54$, NS). (d) For all animals, BLA inactivation increased the amount of time to choose between LR and HR levers (inactivation: $F_{1.12}=17.310$, $p=0.00 \mathrm{I}$; inactivation $\times$ choice: $F_{1,12}=2.210, N S$; group/inactivation $\times$ group: all Fs $<0.322$, NS). Data are shown as the mean percent $(a-c)$ or mean time in seconds (d) for each variable $( \pm$ SEM).

Other behavioral measures. As per physical effort literature (Ghods-Sharifi et al, 2009), inactivation of the BLA increased the amount of time for animals to choose between LR and HR levers (Figure 3d; inactivation: $F_{1,12}=17.310$, $p=0.001$; inactivation $\times$ choice: $\left.\mathrm{F}_{1,12}=2.210, \mathrm{NS}\right)$. This effect was the same for workers and slackers (group/ inactivation $\times$ group: all $\mathrm{Fs}<0.322$, NS), and animals took equally long to choose between LR and HR options (choice/ choice $\times$ group: all Fs $<1.331$, NS). BLA inactivation also increased the latency to make a correct nosepoke response across all trial types (inactivation: $\mathrm{F}_{1,12}=6.261, p=0.028$; inactivation $\times$ choice: $F_{1,12}=0.282$, NS) and correct responses were equally fast for LR and HR trials (choice: $\mathrm{F}_{1,12}=0.017$, NS). As per previous results (Cocker et al, 2012), animals collected rewards faster following HR trials than for LR trials (choice: $F_{1,12}=73.120, p<0.001$ ), and subsequent ANOVAs showed that slackers collected on HR trials faster than workers (group: $\mathrm{F}_{1,12}=5.667, p=0.035$; LR-only group: $\mathrm{F}_{1,12}=1.769$, NS; HR-only group: $\left.\mathrm{F}_{1,12}=9.369, p=0.010\right)$, suggesting that slackers are not simply indifferent to reward magnitude. BLA inactivation had no effect on collection latency (inactivation/ inactivation $\times$ choice: all Fs $<0.689$, NS). Animals failed to respond by nosepoke more often for HR $v s$ LR trials (choice: $\mathrm{F}_{1,12}=11.029, p=0.006$ ) but BLA inactivation had no effect on these response omissions (inactivation/ inactivation $\times$ choice: all $\mathrm{Fs}<1.460$, NS), and there were no differences between workers and slackers revealed by

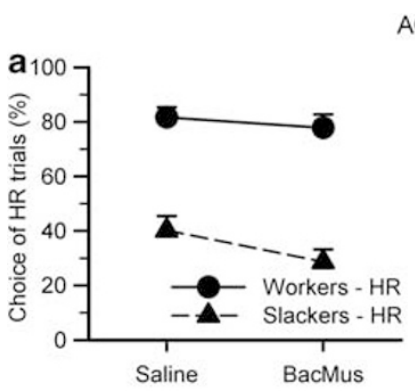

ACC
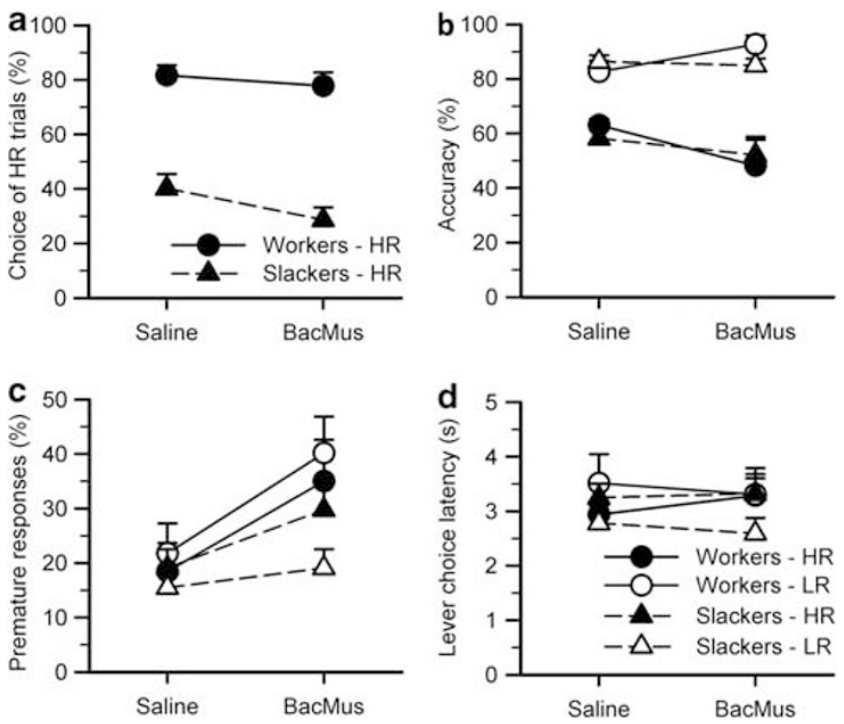

Figure 4 Effects of ACC inactivations on the rodent cognitive effort task (a) Infusion of baclofen-muscimol into the ACC decreased all animals preference for the HR option (inactivation: $F_{1,24}=6.178, p=0.020$; inactivation $\times$ group: $F_{1,24}=1.572$, NS). (b) $A C C$ inactivation had virtually no effect on accuracy, with a significant inactivation by choice interaction but not significantly increasing or decreasing LR or HR when considered alone (inactivation: $F_{1,24}=0.023, N S$; inactivation $\times$ choice: $F_{1,24}=4.671$, $p=0.04 \mathrm{I}$; inactivation $\times$ choice $\times$ grou $p=3.918, p=0.059$; LR, workers only-inactivation: $F_{1,9}=4.977, p=0.053$; LR/HR/HR workers/LR slackers/ HR slackers - inactivation: all Fs <2.962, NS). (c) For all animals, inactivation of the ACC increased premature responding for both trial types (inactivation: $F_{1,24}=9.718, p=0.005$; group/inactivation $\times$ choice/inactivation $\times$ choice $\times$ group: all $F s<1.89$ I, NS). (d) ACC inactivation had no effect on the latency to choose between LR and HR levers (inactivation/ inactivation $\times$ choice: all Fs $<1.938$, NS). Data are shown as the mean percent $(a-c)$ or mean time in seconds $(d)$ for each variable $( \pm$ SEM).

repeated-measures ANOVA (group/inactivation $\times$ group/ choice $\times$ group: all Fs $<2.528$, NS). For all animals, inactivation of the BLA increased the number of lever (choice) omissions (inactivation: $\mathrm{F}_{1,12}=16.740, p=0.001$; inactivation $\times$ group: $F_{1,12}=0.287$, NS; group: $F_{1,12}=0.638$, NS) and decreased the number of trials completed (inactivation: $\mathrm{F}_{1,12}=22.003, p=0.001$; inactivation $\times$ group: $0.004, \mathrm{NS}$ ). Slackers completed more trials than workers when infused with saline but not baclofenmuscimol (group: $\mathrm{F}_{1,12}=5.026, \quad p=0.045$; salineonly group: $\mathrm{F}_{1,12}=11.374, p=0.006$; drug-only group: $\left.\mathrm{F}_{1,12}=1.287, \mathrm{NS}\right)$.

\section{ACC Inactivation}

Choice behavior, accuracy, and premature responses. Unlike the differential effects of BLA inactivation for workers $v s$ slackers, infusion of baclofen-muscimol into the ACC decreased all animals' preference for the HR option (Figure 4a; inactivation: $\mathrm{F}_{1,24}=6.178, p=0.020$; inactivation $\times$ group: $\mathrm{F}_{1,24}=1.572$, NS). ACC inactivation had a stronger effect on slackers when considered across quartiles, but inactivation effects were present for slackers throughout all quartiles of the session, as demonstrated by lack of inactivation $\times$ quartile effect on repeated-measures 
ANOVA (inactivation: $\mathrm{F}_{1,24}=2.710, \mathrm{NS}$; inactivation $\times$ group: $F_{1,24}=4.996, p=0.035 ;$ quartile/quartile $\times$ group/ inactivation $\times$ quartile/inactivation $\times$ quartile $\times$ group: all Fs $<2.710$, NS; workers only-inactivation/quartile/inactivation $\times$ quartile: all Fs $<0.728$, NS; slackers-inactivation: $\mathrm{F}_{1,15}=7.345, p=0.016$; quartile/inactivation $\times$ quartile: all Fs $<1.276, \mathrm{NS})$.

In contrast, ACC inactivation had virtually no effect on accuracy, with a significant inactivation by choice interaction but not significantly increasing or decreasing LR or HR when considered alone (Figure $4 \mathrm{~b}$; inactivation: $\mathrm{F}_{1,24}=0.023$, NS; inactivation $\times$ choice: $\mathrm{F}_{1,24}=4.671, p=0.041$; inactivation $\times$ choice $\times$ group $=3.918, \quad p=0.059 ; \quad$ LR, workers onlyinactivation: $\mathrm{F}_{1,9}=4.977, p=0.053$; LR/HR/HR workers/LR slackers/HR slackers-inactivation: all Fs $<2.962$, NS). As per 5CSRTT literature (Muir et al, 1996), ACC inactivation increased premature responding for both trial types (Figure 4c; inactivation: $\mathrm{F}_{1,24}=9.718, p=0.005$; inactivation $\times$ choice/inactivation $\times$ choice $\times$ group: all Fs $<1.369$, NS). There were no group differences between workers and slackers for accuracy and premature responding (group: all Fs $<1.891$, NS).

Other behavioral measures. Inactivation of the ACC had no effect on the latency to choose between LR and HR levers (Figure 4d; inactivation/inactivation $\times$ choice: all Fs $<1.938$, NS), but increased the time needed to make a correct nosepoke response for both trial types (inactivation: $\mathrm{F}_{1,24}=35.602, p<0.001$; inactivation $\times$ choice: $\mathrm{F}_{1,24}=0.033$, NS). ACC inactivation did not affect the latency to collect reward following a correct response (inactivation/ inactivation $\times$ choice: all Fs $<1.343$, NS) but increased response omissions for all animals (inactivation: $\mathrm{F}_{1,24}=37.344$, $p<0.001$; inactivation $\times$ choice: $\mathrm{F}_{1,24}=3.711, p=0.066$; LRonly inactivation: $\mathrm{F}_{1,24}=22.433, p<0.001$; HR-only inactivation: $\left.\mathrm{F}_{1,24}=34.468, p<0.001\right)$. Inactivation of the ACC also increased the number of lever (choice) omissions (inactivation: $F_{1,24}=5.873, p=0.023$ ) and decreased the number of completed trials (inactivation: $F_{1,24}=31.955$, $p<0.001$ ) for all animals. There were no significant differences between workers and slackers on all of the above measures (group/inactivation $\times$ group/inactivation $\times$ choice $\times$ group: all Fs $<2.811$, NS).

\section{DISCUSSION}

Here we show for the first time that both the BLA and the ACC subserve decision making with cognitive effort costs in rats, as inactivation of either of these regions altered animals' willingness to expend mental effort. Interestingly, a clear dissociation between inactivations of the BLA and ACC was observed. Without intact functioning of the BLA, workers 'slacked off and slackers 'worked harder', whereas loss of ACC function caused all animals to reduce their choice of high-effort trials. These results are in contrast to physical effort decision-making tasks, where loss of either BLA or ACC decreased choice of high-effort options in rats (Floresco and Ghods-Sharifi, 2007; Walton et al, 2003b). The dissociation of these two regions in this study extended beyond choice: BLA inactivations had no effect on motor impulsivity and lengthened the latency to choose between LR and HR options; conversely, ACC inactivations increased motor impulsivity but had no effect on choice latency.

A number of alternate explanations must also be considered for the changes in choice behavior. First, inactivation may have impaired animals' ability to predict reward magnitude and/or difficulty associated with the lever options, rather than altering effortful decision making per se. This interpretation is especially relevant for the BLA inactivation, which had opposing effects on workers' and slackers' choice. If a given inactivation impaired animals' ability to predict levers' costs and benefits, choice should move toward indifference or randomness, that is, $50 \%$ for HR. However, BLA inactivation caused choice behavior to converge on the baseline average for the cohort, well above chance; ACC inactivation, on the other hand, actually caused slackers' choice to move away from indifference. Furthermore, if animals could not predict the trial's reward size, animals would presumably return to the food tray to collect their reward equally fast for LR vs HR trials. In both control and inactivation conditions, however, all animals collected HR reward faster than LR, suggesting that animals could predict the larger, two-pellet reward. Taken together, this evidence would suggest that animals were not indifferent to the outcomes and retained the associated values of options despite their altered choice.

Second, a change in choice behavior may be the result of degraded performance rather than changes in effortful decision making. However, neither inactivation significantly affected accuracy, implying that changes to choice behavior were not due to an inability to allocate visuospatial attention to the task. Relatedly, changes in choice because of inactivation were observed from the outset rather than accumulating across the session.

Third, inactivation of BLA and ACC also produced a range of motor effects, including increased latency to make correct nosepoke responses, increased number of lever (choice) omissions, and decreased number of completed trials. It is possible that these motor effects may have decreased motivation to expend effort, and thus choice of the HR option. However, loss of BLA or ACC function decreases willingness to exert physical effort on a T-maze task, but when a second barrier is placed in the LR arm (thus equalizing effort costs for LR and HR options), rats resume their preference for $\mathrm{HR}$ (Floresco and Ghods-Sharifi, 2007; Walton et al, 2003a). Thus, the effects of BLA and ACC inactivations likely represent a shift in their decision-making processes rather than some general motor impairment.

Fourth, pharmacological inactivations encompass a considerably smaller region of brain mass ( $\sim 1 \mathrm{~mm}$ spread; see Floresco et al, 2006; Marquis et al, 2007) as compared with excitotoxic lesions (Walton et al, 2002) used in some previous studies. Such a spread would be sufficient to inactivate substantial portions of the BLA but likely only equivalent to a partial ACC lesion. Thus, it may be argued that this study's results and, notably, how they differ from previous studies, are due to unintended consequences of experimental design. However, the current methods closely adhered to a physical effort task with BLA inactivations (Ghods-Sharifi et al, 2009). Previous literature noted that loss of BLA function increased latency to choose (Ghods- 
Sharifi et al, 2009), whereas loss of ACC function increased motor impulsivity as measured by premature responding (Muir et al, 1996); critically, both effects were observed in this study. Furthermore, Muir et al (1996) showed that medial prefrontal cortex (mPFC) lesions decreased rats' accuracy on the 5CSRTT, an effect not observed in this study. Thus, it is unlikely that the baclofen-muscimol substantially spread from ACC to surrounding regions such as the mPFC. Altogether, it is reasonable to infer that the differences observed for BLA/ACC inactivations on the rCET $v s$ a T-maze or lever-pressing task were due to the costs (ie, cognitive $v s$ physical effort) and not some artifact of experimental design.

It is also important to consider the prevalence of individual differences on the rCET, especially because of their absence from the physical effort literature. As discussed in much greater length in the initial rCET article (Cocker et al, 2012), variability in choice preference (ie, workers and slackers) appears to reflect differences in sensitivity to the effort costs. Slackers and workers performed the task equally well, as demonstrated by their equal accuracy; this suggests that choice preferences do not simply reflect inferior $v s$ superior attentional performance but rather lower $v s$ higher willingness to expend effort. Nor was choice preference simply driven by probability of reward: a control task was performed in the preliminary cohort, wherein effort costs were removed and control animals were yoked to experimental animals' rates of reward, and these control animals demonstrated substantially different behavior than their experimental counterparts (Cocker et al, 2012). Workers and slackers responded with equal rates of premature responding, suggesting that impulsivity was not responsible for differences in choice. In addition, as described above, both groups of rats appeared to understand the contingencies of the task, as reflected by faster collection of large $v s$ small rewards. The greater variation of choice on the rCET, as compared with physical tasks, may be explained by a greater number of trials completed (and thus a greater total amount of effort exertion per session), a higher level of difficulty ( $\sim 60-70 \%$ accuracy for the rCET, $v s$ virtually guaranteed reward on the physical T-maze, for example), or other differences in task design, rather than simply the type of cost (ie, cognitive $v s$ physical).

Contemporary understanding of amygdalar function has moved away from simple aversive stimulus-outcome associations and toward a role in acquiring, updating, and monitoring value (Everitt et al, 2003; Morrison and Salzman, 2010). Single-cell recordings in primates demonstrate that separate populations of amygdala neurons track positive and negative values of both conditioned and unconditioned stimuli, suggesting that the amygdala encodes a state value for any given moment (Belova et al, 2007, 2008). In rats, BLA neurons fire more robustly for rewarded $v s$ unrewarded stimuli, and BLA inactivation suppresses animals' responding for reinforced cues (Ishikawa et al, 2008). Furthermore, BLA firing precedes and drives activity in the nucleus accumbens (NAc), a critical site for motivated behavior (Ambroggi et al, 2008). Loss of amygdala function in both non-human primates and rats prevents animals from updating their behavior in response to devaluation of a given option via sensoryspecific satiety (Wellman et al, 2005; West et al, 2012); value inflation is also dependent on the BLA (Wassum et al, 2011). Such inflexibility in behavior is also observed in patients with amygdala damage, when a previously beneficial option's associated risk of punishment is increased (Bechara et al, 1999).

As regards effort-based decision making, BLA inactivation decreases preference for high-effort options on both T-maze and operant lever-pressing (ie, physical) paradigms (Floresco and Ghods-Sharifi, 2007; Ghods-Sharifi et al, 2009). This leads to the prima facie assumption that BLA activity overpowers internal representations of cost in order to bias behavior toward highly rewarding options. However, this study suggests a more nuanced contribution of the BLA to effortful decision making. The effects of BLA inactivation on the rCET depended critically on animals' baseline preferences: animals did not uniformly 'slack off in response to BLA inactivation, but rather moved toward the cohort average. This is not simply a statistical regression to the mean, as choice preferences prove remarkably stable within-subjects across baseline and all experimental manipulations (Cocker et al, 2012). Nor are the effects of BLA inactivation because of indifference between the choices, as choice of the HR option remains far above chance. Unlike previous Pavlovian conditioning experiments (Belova et al, 2007), LR and HR stimuli (levers) carry both appetitive (sucrose reward) and aversive (cognitive effort) predictions, and as such may engage both populations of valencespecific neurons in the BLA, which in turn project to regions such as the NAc. The population-level activity of the BLA may therefore represent the interaction of costs and benefits in a choice, encoding the subjective value of given options, rather than the stimulus properties per se; singlecell recordings from human amygdala strongly support this hypothesis (Jenison et al, 2011). As such, BLA inactivations may have fundamentally disrupted rats' subjective values of options on the rCET, and in absence of these subjective weights, the subsequent decisions relied more heavily on objective stimulus properties (Dolan, 2012) or simpler processes such as matching law (Herrnstein, 1970).

Such a hypothesis could also explain why the BLA appears necessary for both cognitive and physical effortbased decision making, but with distinct effects of its inactivation depending on the task costs. In the rCET, the considerable and sustained mental effort costs, in addition to no guarantee of reward, may drive high activity in negatively valenced BLA neurons of some animals, shifting these animals' preference away from high-effort options. By comparison, the relatively brief costs of physical tasks, as well as virtually guaranteed receipt of reward, may allow positively valenced BLA neurons to dominate population activity and guide behavior of most animals toward highly rewarding options.

On the other hand, ACC inactivations decreased willingness to expend cognitive effort for all animals, the same effect observed on physical effort-based decision-making tasks (Rudebeck et al, 2006a; Walton et al, 2003b, 2009). In non-human primates, ACC neurons preferentially track action-outcome $v s$ stimulus-outcome associations (Luk and Wallis, 2013) and appear to encode the subjective sensitivity to reward value following its delivery (Cai and PadoaSchioppa, 2012); this is in contrast to BLA neurons which, as mentioned above, likely represent the subjective 
value of given options before reward delivery. In rats, the ACC shows greater metabolic demands when integrating effort costs and reward magnitude $v s$ when effort costs are held constant and only reward magnitude varies (Endepols et al, 2010). Similarly in humans, functional imaging studies suggest that ACC activity at choice and feedback correlates with the option that maximizes reward over the long term (Boorman et al, 2013), and ACC activation is greater when rejecting highly valued options $v s$ rejecting less preferred options (Izuma et al, 2010). Altogether, this suggests that the ACC biases individuals away from prepotent but suboptimal responses, in effect guiding behavior toward maximal returns. Such a putative function explains why ACC inactivation shifted all animals' preference toward the LR option on the rCET, and why it would have a stronger effect on slackers $v s$ workers, namely the already strong drive for slackers to choose LR trials. This function also corresponds well with tobacco- and alcohol-dependent individuals, wherein ACC dysfunction heightens salient, prepotent cues for drug, and can impair decision making (Janes et al, 2013; Le Berre et al, 2012).

The disturbances of effort-based decision making observed in a number of mental health populations (Gold et al, 2013; Treadway et al, 2012a) may therefore reflect underlying functional and connective changes to regions such as the amygdala and ACC. Dopamine function, which has long been implicated in willingness to expend effort (Salamone, 2009; Salamone et al, 2007; Treadway et al, 2012b), may also act on these regions to affect effortful decision making (Schweimer and Hauber, 2006; Schweimer et al, 2005), and thus its contribution to cognitive effort is currently under study. The overlapping-yet-distinct effects observed in the present experiments suggest that regions not required for physical effort-based decision making, such as the prefrontal cortex, may in fact be necessary for decision making with cognitive effort costs (Schmidt et al, 2012), and the NAc' prevalence in the effort literature warrants future consideration of its involvement in choice on the rCET. To conclude, these results may disentangle the unique contributions of the BLA and ACC, namely the subjective valuation of options $v s$ the biasing of behavior toward advantageous choice strategies, respectively, and offer unique insights into targeting these regions for therapeutic intervention.

\section{FUNDING AND DISCLOSURE}

This work was supported by a discovery grant awarded to CAW from the Canadian Natural Sciences and Engineering Council. CAW also receives salary support through the Michael Smith Foundation for Health Research and the Canadian Institutes of Health Research (CIHR) New Investigator Award program. JGH was supported by a CIHR Doctoral Research Award. The authors declare no conflict of interest.

\section{REFERENCES}

Ambroggi F, Ishikawa A, Fields HL, Nicola SM (2008). Basolateral amygdala neurons facilitate reward-seeking behavior by exciting nucleus accumbens neurons. Neuron 59: 648-661.
Basten U, Biele G, Heekeren HR, Fiebach CJ (2010). How the brain integrates costs and benefits during decision making. Proc Natl Acad Sci USA 107: 21767-21772.

Bechara A, Damasio H, Damasio AR, Lee GP (1999). Different contributions of the human amygdala and ventromedial prefrontal cortex to decision-making. J Neurosci 19: 5473-5481.

Belova MA, Paton JJ, Morrison SE, Salzman CD (2007). Expectation modulates neural responses to pleasant and aversive stimuli in primate amygdala. Neuron 55: 970-984.

Belova MA, Paton JJ, Salzman CD (2008). Moment-tomoment tracking of state value in the amygdala. J Neurosci 28: 10023-10030.

Boorman ED, Rushworth MF, Behrens TE (2013). Ventromedial prefrontal and anterior cingulate cortex adopt choice and default reference frames during sequential multi-alternative choice. J Neurosci 33: 2242-2253.

Brand M, Grabenhorst F, Starcke K, Vandekerckhove MM, Markowitsch HJ (2007). Role of the amygdala in decisions under ambiguity and decisions under risk: evidence from patients with Urbach-Wiethe disease. Neuropsychologia 45: 1305-1317.

Cai X, Padoa-Schioppa C (2012). Neuronal encoding of subjective value in dorsal and ventral anterior cingulate cortex. $J$ Neurosci 32: 3791-3808.

Cocker PJ, Hosking JG, Benoit J, Winstanley CA (2012). Sensitivity to cognitive effort mediates psychostimulant effects on a novel rodent cost/benefit decision-making task. Neuropsychopharmacology 37: 1825-1837.

Croxson PL, Walton ME, O'Reilly JX, Behrens TE, Rushworth MF (2009). Effort-based cost-benefit valuation and the human brain. J Neurosci 29: 4531-4541.

Dolan RJ (2012). Neuroscience of Preference and Choice: Cognitive and Neural Mechanisms. 1st edn. Academic Press/Elsevier: London; Waltham, MA, xiv, 342p.

Egeland J, Johansen SN, Ueland T (2010). Do low-effort learning strategies mediate impaired memory in ADHD? J Learn Disabil 43: $430-440$.

Endepols H, Sommer S, Backes H, Wiedermann D, Graf R, Hauber W (2010). Effort-based decision making in the rat: an [18F]fluorodeoxyglucose micro positron emission tomography study. J Neurosci 30: 9708-9714.

Everitt BJ, Cardinal RN, Parkinson JA, Robbins TW (2003). Appetitive behavior: impact of amygdala-dependent mechanisms of emotional learning. Ann N Y Acad Sci 985: 233-250.

Floresco SB, Ghods-Sharifi S (2007). Amygdala-prefrontal cortical circuitry regulates effort-based decision making. Cerebral Cortex 17: $251-260$.

Floresco SB, Ghods-Sharifi S, Vexelman C, Magyar O (2006). Dissociable roles for the nucleus accumbens core and shell in regulating set shifting. J Neurosci 26: 2449-2457.

Floresco SB, St Onge JR, Ghods-Sharifi S, Winstanley CA (2008a). Cortico-limbic-striatal circuits subserving different forms of cost-benefit decision making. Cogn Affect Behav Neurosci 8: 375-389.

Floresco SB, Tse MT, Ghods-Sharifi S (2008b). Dopaminergic and glutamatergic regulation of effort- and delay-based decision making. Neuropsychopharmacology 33: 1966-1979.

Ghods-Sharifi S, St Onge JR, Floresco SB (2009). Fundamental contribution by the basolateral amygdala to different forms of decision making. J Neurosci 29: 5251-5259.

Gold JM, Strauss GP, Waltz JA, Robinson BM, Brown JK, Frank MJ (2013). Negative symptoms of schizophrenia are associated with abnormal effort-cost computations. Biol Psychiatry 74: 130-136.

Hammar A, Strand M, Ardal G, Schmid M, Lund A, Elliott R (2011). Testing the cognitive effort hypothesis of cognitive impairment in major depression. Nord J Psychiatry 65: $74-80$. 
Herrnstein RJ (1970). On the law of effect. J Exp Anal Behav 13: 243-266.

Ishikawa A, Ambroggi F, Nicola SM, Fields HL (2008). Contributions of the amygdala and medial prefrontal cortex to incentive cue responding. Neuroscience 155: 573-584.

Izuma K, Matsumoto M, Murayama K, Samejima K, Sadato N, Matsumoto K (2010). Neural correlates of cognitive dissonance and choice-induced preference change. Proc Natl Acad Sci USA 107: 22014-22019.

Janes AC, Jensen JE, Farmer SL, Frederick BD, Pizzagalli DA, Lukas SE (2013). GABA levels in the dorsal anterior cingulate cortex associated with difficulty ignoring smoking-related cues in tobacco-dependent volunteers. Neuropsychopharmacology 38: 1113-1120.

Jenison RL, Rangel A, Oya H, Kawasaki H, Howard MA (2011). Value encoding in single neurons in the human amygdala during decision making. J Neurosci 31: 331-338.

Kool W, McGuire JT, Rosen ZB, Botvinick MM (2010). Decision making and the avoidance of cognitive demand. J Exp Psychol Gen 139: 665-682.

Le Berre AP, Rauchs G, La Joie R, Mezenge F, Boudehent C, Vabret $\mathrm{F}$ et al (2012). Impaired decision-making and brain shrinkage in alcoholism. Eur Psychiatry S0924-9338: 00136-00138.

Luk CH, Wallis JD (2013). Choice coding in frontal cortex during stimulus-guided or action-guided decision-making. J Neurosci 33: $1864-1871$.

Manes F, Sahakian B, Clark L, Rogers R, Antoun N, Aitken M et al (2002). Decision-making processes following damage to the prefrontal cortex. Brain 125(Pt 3): 624-639.

Marquis JP, Killcross S, Haddon JE (2007). Inactivation of the prelimbic, but not infralimbic, prefrontal cortex impairs the contextual control of response conflict in rats. Eur J Neurosci 25: 559-566.

McGuire JT, Botvinick MM (2010). Prefrontal cortex, cognitive control, and the registration of decision costs. Proc Natl Acad Sci USA 107: 7922-7926.

Morrison SE, Salzman CD (2010). Re-valuing the amygdala. Curr Opin Neurobiol 20: 221-230.

Muir JL, Everitt BJ, Robbins TW (1996). The cerebral cortex of the rat and visual attentional function: dissociable effects of mediofrontal, cingulate, anterior dorsolateral, and parietal cortex lesions on a five-choice serial reaction time task. Cereb Cortex 6: 470-481.

Naccache L, Dehaene S, Cohen L, Habert MO, Guichart-Gomez E, Galanaud D et al (2005). Effortless control: executive attention and conscious feeling of mental effort are dissociable. Neuropsychologia 43: 1318-1328.

Paxinos G, Watson C (1998). The Rat Brain in Stereotaxic Coordinates. 4th edn. Academic Press: San Diego, xxvi, [237] p of platespp.

Randall PA, Pardo M, Nunes EJ, Lopez Cruz L, Vemuri VK, Makriyannis A et al (2012). Dopaminergic modulation of effortrelated choice behavior as assessed by a progressive ratio chow feeding choice task: pharmacological studies and the role of individual differences. PLoS ONE 7: e47934.

Robbins TW (2002). The 5-choice serial reaction time task: behavioural pharmacology and functional neurochemistry. Psychopharmacology (Berl) 163: 362-380.

Rudebeck PH, Walton ME, Smyth AN, Bannerman DM, Rushworth MF (2006a). Separate neural pathways process different decision costs. Nat Neurosci 9: 1161-1168.

Rudebeck PH, Walton ME, Smyth AN, Bannerman DM, Rushworth MFS (2006b). Separate neural pathways process different decision costs. Nat Neurosci 9: 1161-1168.

Salamone JD (2009). Dopamine, effort, and decision making: theoretical comment on Bardgett et al (2009). Behav Neurosci 123: $463-467$.
Salamone JD, Correa M, Farrar A, Mingote SM (2007). Effortrelated functions of nucleus accumbens dopamine and associated forebrain circuits. Psychopharmacology (Berl) 191: 461-482.

Salamone JD, Cousins MS, Bucher S (1994). Anhedonia or anergia? Effects of haloperidol and nucleus accumbens dopamine depletion on instrumental response selection in a T-maze cost/ benefit procedure. Behav Brain Res 65: 221-229.

Schmidt L, Lebreton M, Clery-Melin ML, Daunizeau J, Pessiglione M (2012). Neural mechanisms underlying motivation of mental versus physical effort. PLoS Biol 10: e1001266.

Schweimer J, Hauber W (2006). Dopamine D1 receptors in the anterior cingulate cortex regulate effort-based decision making. Learn Mem 13: 777-782.

Schweimer J, Saft S, Hauber W (2005). Involvement of catecholamine neurotransmission in the rat anterior cingulate in effortrelated decision making. Behav Neurosci 119: 1687-1692.

Smith BW, Mitchell DG, Hardin MG, Jazbec S, Fridberg D, Blair RJ et al (2009). Neural substrates of reward magnitude, probability, and risk during a wheel of fortune decision-making task. Neuroimage 44: 600-609.

Sokol-Hessner P, Camerer CF, Phelps EA (2013). Emotion regulation reduces loss aversion and decreases amygdala responses to losses. Social Cognitive Affective Neurosci 8: 341-350.

Treadway MT, Bossaller NA, Shelton RC, Zald DH (2012a). Effort-based decision-making in major depressive disorder: a translational model of motivational anhedonia. J Abnorm Psychol 121: 553-558.

Treadway MT, Buckholtz JW, Cowan RL, Woodward ND, Li R, Ansari MS et al (2012b). Dopaminergic mechanisms of individual differences in human effort-based decision-making. J Neurosci 32: 6170-6176.

van Honk J, Eisenegger C, Terburg D, Stein DJ, Morgan B (2013). Generous economic investments after basolateral amygdala damage. Proc Natl Acad Sci USA 110: 2506-2510.

Walton ME, Bannerman DM, Alterescu K, Rushworth MF (2003a). Functional specialization within medial frontal cortex of the anterior cingulate for evaluating effort-related decisions. J Neurosci 23: 6475-6479.

Walton ME, Bannerman DM, Alterescu K, Rushworth MFS (2003b). Functional specialization within medial frontal cortex of the anterior cingulate for evaluating effort-related decisions. J Neurosci 23: 6475-6479.

Walton ME, Bannerman DM, Rushworth MFS (2002). The role of rat medial frontal cortex in effort-based decision making. J Neurosci 22: 10996-11003.

Walton ME, Devlin JT, Rushworth MF (2004). Interactions between decision making and performance monitoring within prefrontal cortex. Nat Neurosci 7: 1259-1265.

Walton ME, Groves J, Jennings KA, Croxson PL, Sharp T, Rushworth MF et al (2009). Comparing the role of the anterior cingulate cortex and 6-hydroxydopamine nucleus accumbens lesions on operant effort-based decision making. Eur J Neurosci 29: 1678-1691.

Wassum KM, Cely IC, Balleine BW, Maidment NT (2011). Micro-opioid receptor activation in the basolateral amygdala mediates the learning of increases but not decreases in the incentive value of a food reward. J Neurosci 31: 1591-1599.

Wellman LL, Gale K, Malkova L (2005). GABAA-mediated inhibition of basolateral amygdala blocks reward devaluation in macaques. J Neurosci 25: 4577-4586.

West EA, Forcelli PA, Murnen AT, McCue DL, Gale K, Malkova L (2012). Transient inactivation of basolateral amygdala during selective satiation disrupts reinforcer devaluation in rats. Behav Neurosci 126: 563-574.

Williams ZM, Bush G, Rauch SL, Cosgrove GR, Eskandar EN (2004). Human anterior cingulate neurons and the integration of monetary reward with motor responses. Nat Neurosci 7: 1370-1375. 
Winstanley CA, Chudasama Y, Dalley JW, Theobald DE, Glennon JC, Robbins TW (2003). Intra-prefrontal 8-OH-DPAT and M100907 improve visuospatial attention and decrease impulsivity on the five-choice serial reaction time task in rats. Psychopharmacology (Berl) 167: 304-314.

Winstanley CA, Zeeb FD, Bedard A, Fu K, Lai B, Steele C et al (2010). Dopaminergic modulation of the orbitofrontal cortex affects attention, motivation and impulsive responding in rats performing the five-choice serial reaction time task. Behav Brain Res 210: 263-272.

Zeeb FD, Robbins TW, Winstanley CA (2009). Serotonergic and dopaminergic modulation of gambling behavior as assessed using a novel rat gambling task. Neuropsychopharmacology 34: 2329-2343. 